\title{
SYNTHESIS AND CHARACTERIZATION OF POLY(METHYL METHACRYLATE)-SILICA, -ALUMINA AND -SILICA-ALUMINA SOL-GEL NANOCOMPOSITES
}

\author{
F. ORELLANA ${ }^{1, *}$, J. LISPERGUER ${ }^{l}$ AND A. PEREZ-GUERRERO ${ }^{2}$ \\ ${ }^{\prime}:$ Depto. Química, Facultad de Ciencias, Universidad del Bio-Bio, Av. Collao 1202-Concepción-CHILE. \\ 2 : Centro de Investigación de Polímeros avanzados (CIPA) - CHILE
}

(Received: August 19, 2010 - Accepted: March 16, 2011)

\begin{abstract}
The development of nanostructructured polymethilmethacrylate (PMMA) surfaces was studied by the incorporation of silica, alumina and silica-alumina, the former obtained by Sol-gel procedure. The materials were obtained in a polymer solution (acetone, isopropanol) and contains variable amounts of the inorganic component (10 and $20 \%$ in the mass). The SEM studies show the modification of the polymer structure by the addition of the silicon oxide and aluminum oxide; though not all the samples show a high dispersion of the inorganic component. The FT-IR spectrum showed a reduction in the intensity of the carbonyl signal associated to the polymer, due to the incorporation of the filler and the presence of bands attributed to the inorganic components incorporated. Through TGA and DSC studies the modification of the thermal stability of the polymer was proved, depending on the inorganic component used and its composition.
\end{abstract}

\section{INTRODUCTION}

For over a decade the polymers industry and the academic research laboratories have been paying attention to inorganic polymer -particle nanocomposites of a very small size (1-2). With these materials it has been possible to modify the properties of the polymer by adding specific fillers that are distributed at a nanometric level inside the polymeric matrix. They offer the combined advantages of polymer compounds, flexibility, ductility and processing capacity, as well as completely new properties, stiffness and high thermal stability of the nanostructured materials (3-5). The reduction in size of the structure of materials (nanotechnology) involves numberless design possibilities. It allows to develop customized plastic materials for specific applications and optimize the properties of a polymer. The choice of the initial materials and the polymer properties desired to boost are what inspires the investigation of the composites (6). The polymer nanocomposites contain a filler, usually clay, of nanometric dimension that spreads through the polymer. Its content can not exceed $20 \%$ in mass (7). The dispersion of the inorganic component in the polymer may be at molecular or agglomerate level, depending on the interaction area of both components (8).

The sol-gel procedure offers an hydrolytic route at ambient temperature to obtain metal oxides ( $\mathrm{Si}, \mathrm{Al}, \mathrm{Ti}, \mathrm{Zr}$, etc.) in nanostructured matrices (9). These small molecules can be trapped in polymer networks and retain its chemical properties. The conditions of the sol-gel process where $\mathrm{SiO}_{2}, \mathrm{Al}_{2} \mathrm{O}_{3}$, etc, reticules are formed, condition the final structure of the respective oxide : the condensation degree, the intermediate linear species before a complex one, the average particle size, etc. Some of the fundamental parameters that control the topography of these oxides in composite materials derived from solgel reactions include the nature of the catalyst used to increase the reactivity of the alkoxide (as well as its amount, $\mathrm{pH}$ ), the water available to hydrolyze the oxide precursor (related to the stoichiometric amount needed to hydrolyze completely a molecule of the oxide precursor) and the relation between the percentages of the organic and inorganic phases in the final hybrid material. The first parameter (catalyst) and the second (water) were established in this work to synthesize materials of the size of silica and alumina around the tens of nanometers.

The final properties of the composite material are more complex than the total of the properties of both components, due to synergic effects caused by the heterogeneity that occurs at nanoscale levels, where the interactions of the phases are maximized by the high surface area of the inorganic component $(10,11)$. The sol-gel procedure besides producing the inorganic component with suitable properties for the interaction with the polymer, easy the incorporation of the inorganic component into the organic one due to the solft conditions used in the preparation. The final material contains inorganic structures interlocked or substituted by organic groups of the polymer. The nature of the interphase between the organic and inorganic component may be due to the interactions caused by the van der Waals strengths, by H-bond or electrostatic forces; or the components are unified by a strong chemical bond (covalent or ionic-covalent).

The polymethylmethacrilate (PMMA) is an important optic thermoplastic material with perfect transparency in the visible region, it is mostly used in optics and electronics; but its application is limited by the low thermal stability of this polymer (12). It has been managed to obtain PMMA-titania nanocomposites by the sol -gel method, but to obtain it requires the use of chelating agents which reduce the flexibility of the nanocomposite material, affecting the thermal behavior and its processing (13).

In this work the formation of a nanocomposite material made of PMMA and silica, alumina or silica-alumina is studied as a filler or booster, the latter obtained by a polymerization through a sol-gel reaction. The amount of silica (tetraethoxysilane, TEOS) and alumina (Secbutoxide of aluminio, SBA) precursor is estimated by assuming a complete hidrosys reaction. This is ensured by preparing the composites at a acid $\mathrm{pH}$. The organic phase is found in a solution and equal amounts of water as for the stoichiometric for SBA are used, and higher for TEOS. To characterize the physical and chemical properties of the nanocomposites materials scanning microscope electron (SEM), infrared spectroscopy (FT-IR), differential scanning calorimetry (DSC) and thermogravimetry (TGA) techniques were used.

\section{EXPERIMENTAL}

Nanocomposites preparation

Silica, alumina and silica-alumina are obtained from an alcohol solution (ethanol and secbutanol) of the precursors TEOS (Aldrich Chem.) and SBA (Sigma Aldric), the homogenization of this mix is achieved by agitation at $40{ }^{\circ} \mathrm{C}$. The reaction of TEOS and SBA was catalyzed in an acid solution, maintaining the molar ratio $\mathrm{HCl} /$ precursor equal to $1.85 \cdot 10^{-2}$ and the ratio $\mathrm{H}_{2} \mathrm{O}$ / precursor equal to 10 for TEOS and 3 for SBA. The content of $\mathrm{SiO}_{2}, \mathrm{Al}_{2} \mathrm{O}_{3}$ and $\mathrm{SiO}_{2}-\mathrm{Al}_{2} \mathrm{O}_{3}$ was 10 and $20 \%$ in weight, assuming that the sol-gel reaction is complete. After 1 hour the polymethyl methacrylate is added (Aldrich, $\mathrm{MW}=40000$ aprox.) dissolved in acetone and the mix is energetically agitated at $40^{\circ} \mathrm{C}$. At first the mix is transparent and then it turns muddy as the solvents are eliminated. The product obtained is dried at $70{ }^{\circ} \mathrm{C}$ for 8 hours and is kept in the desiccator for analysis. Table 1 shows the prepared nanocomposites. 
Table 1. Summary of PMMA-silica, PMMA-alumina y PMMA-silicaalumina samples prepared.

\begin{tabular}{|l|c|c|}
\hline \multicolumn{1}{|c|}{ Nanocomposite } & $\%$ weight filler & $\begin{array}{c}\text { Solvent of } \\
\text { PMMA }\end{array}$ \\
\hline PMMA $_{\text {(acetone) }}-10-\mathrm{SiO}_{2}$ & $10 \% \mathrm{SiO}_{2}$ & Acetone \\
\hline PMMA $_{\text {(acetone) }}-20-\mathrm{SiO}_{2}$ & $20 \% \mathrm{SiO}_{2}$ & Acetone \\
\hline PMMA $_{\text {(isopropanol) }}-10-\mathrm{SiO}_{2}$ & $10 \% \mathrm{SiO}_{2}$ & Isopropanol \\
\hline PMMA $_{\text {(isopropanol) }}-20-\mathrm{SiO}_{2}$ & $20 \% \mathrm{SiO}_{2}$ & Isopropanol \\
\hline PMMA-10- $\mathrm{Al}_{2} \mathrm{O}_{3}$ & $10 \% \mathrm{Al}_{2} \mathrm{O}_{3}$ & Acetone \\
\hline PMMA-20- $\mathrm{Al}_{2} \mathrm{O}_{3}$ & $20 \% \mathrm{Al}_{2} \mathrm{O}_{3}$ & Acetone \\
\hline PMMA-10- $-\mathrm{SiO}_{2}-\mathrm{Al}_{2} \mathrm{O}_{3}$ & $5 \% \mathrm{SiO}_{2}-5 \% \mathrm{Al}_{2} \mathrm{O}_{3}$ & Acetone \\
\hline PMMA-20- $\mathrm{SiO}_{2}-\mathrm{Al}_{2} \mathrm{O}_{3}$ & $10 \% \mathrm{SiO}_{2}-10 \% \mathrm{Al}_{2} \mathrm{O}_{3}$ & Acetone \\
\hline
\end{tabular}

Characterization of the nanocomposites

TGA analysis was used to study the thermal stability of nanocomposites and to confirm the filler final content. A Mettler Toledo Analyzer (Model SDTA 851) was used under $50 \mathrm{~mL} / \mathrm{min}$ nitrogen flow at a warming speed of 10 ${ }^{\circ} \mathrm{C} / \mathrm{min}$, starting from $25^{\circ} \mathrm{C}$ up to $600^{\circ} \mathrm{C}$.

Differential Scanning Calorimetry (DSC) was performed in a Mettler Toledo (Model DSC 822). The nitrogen flow used is $30 \mathrm{~mL} / \mathrm{min}$. The glass-like transition temperature $\left(\mathrm{T}_{\mathrm{g}}\right)$ of the samples was determined in the intersection of the extrapolation of the viscoelastic and glass-like lines (on set temperature). Crystallinity was analyzed by means of isothermal heating at $200{ }^{\circ} \mathrm{C}$ for $5 \mathrm{~min}$ to melt the samples and then a dynamic cooling down from $200{ }^{\circ} \mathrm{C}$ to room temperature at $5{ }^{\circ} \mathrm{C} / \mathrm{min}$. The typical exothermal of crystallization phenomenon was not detected.

The infrared absorption spectrum was obtained in a Nicolett FT-IR (model Nexus) at ambient temperature between 4000 and $400 \mathrm{~cm}^{-1}$, pellets with $\mathrm{KBr}$ were used as a support of the samples.

The SEM micrographs were obtained out of a JEOL microscope (Model $\mathrm{jfm} 6380 \mathrm{lv}$ ). The samples were gold-leaved to obtain the electronic images and for the microanalysis. In this former case, the analysis the gold signals and those of another metal different to what was studied was corrected.

\section{RESULTS AND DISCUSSION}

PMMA has carbonyl groups that can form hydrogen bonds with the $\mathrm{OH}$ groups of Silica and Alumina. The inorganic components obtained must have a very small size (a few nanometers), typical of the sol-gel preparation catalyzed by acid. The thermal behavior of the nanocomposite depends on the matrix of the polymer and on its chemical and morphological characteristics. The change observed by the preparation of the nanocomposites indicate that the polymer spheres turn into an homogenous mass inside of which the nanoparticles of silica and alumina are inlayed. The SEM images (Figure 1) of these do not reveal characteristics that may enable to conclude the type of interaction among both components. However, silica and alumina are easily detected because they provide areas of a different tone than the nanocomposite. The formation of silica or alumina crystallites are found in the surface of the polymer; only in the nanocomposite obtained by dissolution of PMMA in acetone and silica it is possible to see an exfoliated structure or laminar. When the load of silica and alumina increases, multiple layers are formed generating agglomerates.

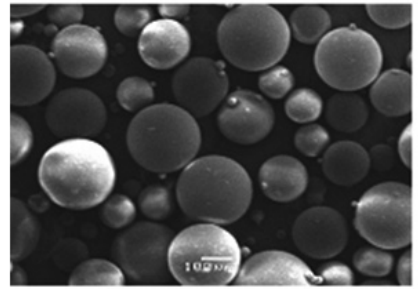

PMMA

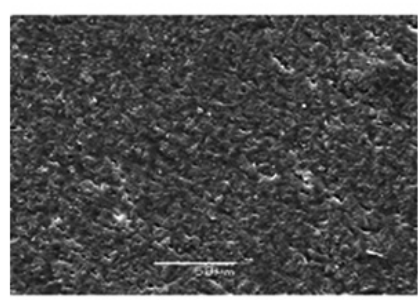

PMMA-10- $\mathrm{Al}_{2} \mathrm{O}_{3}$

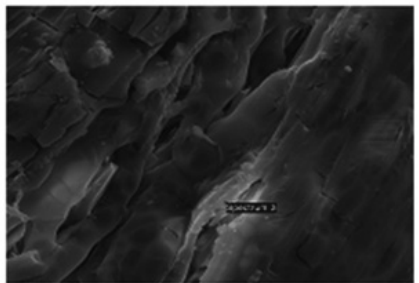

$\mathrm{PMMA}_{\text {(acetons) }}-10-\mathrm{SiO}_{2}$

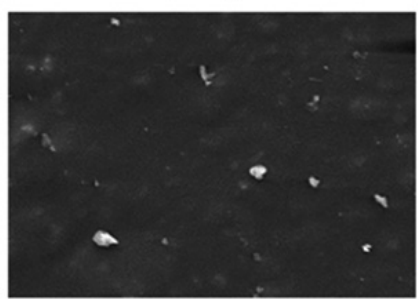

PMMA-10- $\mathrm{SiO}_{2}-\mathrm{Al}_{2} \mathrm{O}_{3}$
Fig. 1. SEM images of polymethyl methacrilate and nanocomposites.

The micrographs of PMMA-SiO, $\mathrm{PMMA}_{2} \mathrm{Al}_{2} \mathrm{O}_{3}$ and $\mathrm{PMMA}-\mathrm{SiO}_{2}-\mathrm{Al}_{2} \mathrm{O}_{3}$ show particles of silica and alumina dispersed inside the polymer matrices. In the virgin polymer it is observed that the distribution of the particle size is not uniform, it fluctuates between 5-30 $\mu \mathrm{m}$, the porous nature of the polymer disappears in nanocomposites. The different appearance in the polymer structure indicates that the inorganic nanoparticles are interacting with the polymer matrix. An homogeneous film of the polymer is formed, and the lighter area correspond to the inorganic component, which adheres to the polymer through the $\mathrm{OH}$ groups of these, in an irregular manner, which was proved by the analysis performed to the micrographs. In the PMMA $\mathrm{SiO}_{2}$ micrograph there were found areas with variable percentages of silica in mass, $0.52 \%, 0.75 \%, 5.13 \%$ and $2.60 \%$. During the preparation of the nanocomposite, the silica and alumina particles must migrate of the acetonePMMA interphase, due to the van der Waals attractions. The heterogeneity in the distribution of the inorganic component is associated to irregularities in this migration and in the polymer surface. The use of isoporpanol as a solvent for the polymer, made it easier for the silica to migrate, since its dispersion is more homogenous in these nanocomposite.

As observed in the SEM micrograph y of PMMA- $\mathrm{Al}_{2} \mathrm{O}_{3}$, the nanoparticles of alumina are dispersed more uniformly in PMMA. As reported by Ash (14) the uniformity of the alumina dispersion is because in these nanocomposites the PMMA reacts with the alumina surface through and hydrolization of the ester bond in the polymeric chain, increasing the degree of interaction among them. It may be attributed to this strong chemisorption the fractured surface observed in the micrography of these nanocomposites. The shape of alumina crystal is spheric and its size is very small, typical of a sol-gel preparation. In the nanocomposites PMMA-SiO $-\mathrm{Al}_{2} \mathrm{O}_{3}$ a more heterogeneous distribution is observed in the crystals size, there are silica-alumina agglomerates formed, which reflect a poor dispersion of the inorganic component. The chemical analysis of agglomerates indicated that there are areas in which Si prevails and in others Al prevails.

TGA curves of nanocomposites in nitrogen indicated that the thermal stability of PMMA was improved by the addition of silica, alumina and silica-alumina fractions, as shown on Figure 2. The thermal decomposition of PMMA shows an small and gradual weight loss (less than $2 \%$ ) up to $300^{\circ} \mathrm{C}$, above that, there is an abrupt reduction in weight at an small range 380-390 ${ }^{\circ} \mathrm{C}$. The thermal decomposition of PMMA is cause mainly by the break down of C-C bonds; it begins by the decomposition of head-head bonds, by the ends vinyl groups and by the random split of polymeric chains (15). 

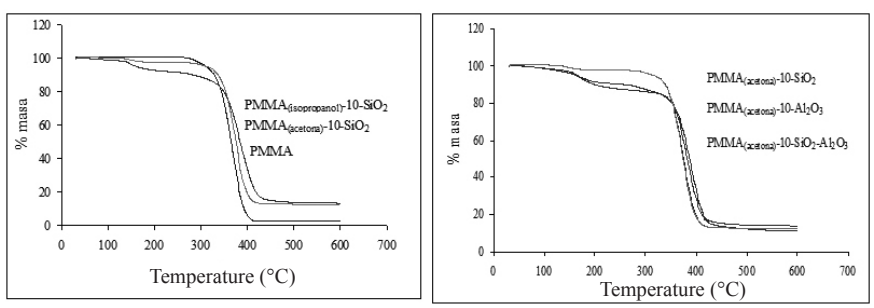

Fig. 2. TGA curves of PMMA and nanocomposites

The polymer TGA shows that there is a gradual reduction in weight when temperature increases, reaching $97 \%$ of weight loss. The remaining 3 $\%$ corresponds to char. The thermogram of the nanocomposites shows two weight loss stages. In stage 1 the degradation of the ends of the polymer occurs, and the elimination of solvent residues. In this stage, silica provides a higher stability to the polymer. The solubility effect of PMMA was studied in another solvent (isobutanol) for its preparation; however, there were not significant differences observed in the characterization of nanocomposites, since there were no improvements in the thermal stability of these. As shown in Table 2 , the presence of $\mathrm{Al}$ and $\mathrm{Si}$ oxides increases up to $20^{\circ} \mathrm{C}$. the temperature at which $50 \%$ of weight loss has occurred $\left(\mathrm{T}_{50^{\circ}}\right)$, compared to the pure PMMA polymer $\left(360^{\circ} \mathrm{C}\right)$.

Table 2. Information of the thermal degradation of nanocomposites obtained by TGA

\begin{tabular}{|c|c|c|c|}
\hline Nanocomposite & Stage 1 & Stage 2 & $\mathrm{~T}_{50 \%}\left({ }^{\circ} \mathrm{C}\right)$ \\
\hline PMMA $_{\text {(acetone) }}-10-\mathrm{SiO}_{2}$ & $2,9 \%$ & $84,6 \%$ & 375 \\
\hline $\mathrm{PMMA}_{\text {(acetone) }}-20-\mathrm{SiO}_{2}$ & $1,8 \%$ & $65,3 \%$ & 387 \\
\hline $\mathrm{PMMA}_{\text {(isopropanol) }}-10-\mathrm{SiO}_{2}$ & $7,7 \%$ & $78,6 \%$ & 384 \\
\hline PMMA-10- $\mathrm{Al}_{2} \mathrm{O}_{3}$ & $12,7 \%$ & $75,5 \%$ & 382 \\
\hline PMMA-10- $\mathrm{SiO}_{2}-\mathrm{Al}_{2} \mathrm{O}_{3}$ & $9,3 \%$ & $76,0 \%$ & 387 \\
\hline
\end{tabular}

In the second stage, at which a break down of the polymer chain occurs, it is observed a greater effect of the incorporation of silica and alumina, the percentage of weight loss is lower. The char percentage increases after warming, this definitely reflects the positive effect in thermal stability. The results show that the improved stability depends on the content of the inorganic component introduced. As expected, the presence of the inorganic component reduces considerably the thermal degradation of PMMA polymer.

The FT-IR spectrum of the polymer showed details of its functional groups. A sharp and intense peak at $1731 \mathrm{~cm}^{-1}$ due to the vibration of the carbonyl group. The intensity of this vibration is reduced with the inorganic component content added to the nanocomposite, which enables to conclude that it is thought these functional groups of PMMA that both components interact. A wide peak between 1260 and $1000 \mathrm{~cm}^{-1}$ attributed to the vibration of the stretching of the $\mathrm{C}-\mathrm{O}$ group in ester bonds. Sharp band and medium intensity between 650 and $500 \mathrm{~cm}^{-1}$ and wide band and less intense between $3200-2900 \mathrm{~cm}^{-1}$ attributed to bending and stretching of C-H (16).

The FT-IR spectrum of the nanocomposites are similar among them. They verily exhibit the absorption of the inorganic groups, maybe because of their low presence in the PMMA matrix. The absence of bands besides those attributed to PMMA in the spectrum, and that most of them remain undisturbed, indicate the purity of the polymer and the formation of the nanocomposite. In general, all of them show a wide absorption band in the area $3600-3000 \mathrm{~cm}^{-1}$ that is attributed to the $\mathrm{OH}$ groups, due to the water that may have been retained and the $\mathrm{Al}-\mathrm{OH}$ and $\mathrm{Si}-\mathrm{OH}$ groups that did not react with the carbonyls of the PMMA. The water retention was prove $\mathrm{d}$ by the medium intensity band that appeared at $1610 \mathrm{~cm}^{-1}$ which is associated to the vibration of its flexion (17). The intensity and sharpness of these signals fluctuate with the different oxides used to prepare the nanocomposites. Given that the intensity of the signal attributed to the carbonyl group is reduced in the nanocomposites, specially at a higher inorganic compound content, it is concluded that the interaction of both phases is through this group of the polymer.

In the nanocomposites spectrum, though the PMMA bands are also found in the same frequency regions as in silica and alumina, there are additional bands attributed to these components. It was possible to assign some of them because they are wider and weak in PMMA; however, they are more intense and sharp in the nanocomposites. In PMMA-SiO there are some frequencies typical to silica at 1100,800 and $460 \mathrm{~cm}^{-1}$ due to the vibrations Si-O-Si and Si$\mathrm{OH}(18,19)$. In $\mathrm{PMMA}-\mathrm{Al}_{2} \mathrm{O}_{3}$ there are some signals observed that may indicate the presence of this oxide, such as the signals found at 640,602 and $453 \mathrm{~cm}^{-1}$ (20). In PMMA-SiO $-\mathrm{Al}_{2} \mathrm{O}_{3}$ the bands were not clearly identified at 2965 and $1601 \mathrm{~cm}^{-1}$, attributed to Si-O-Al (21), thus it was impossible to establish the connection structure between the silica and alumina components. In spite of the fact that the analysis of the SEM micrographs does show the presence of silica and alumina in the agglomerates analyzed, and there is a modification of the distribution of alumina observed by the presence of silica.

The increment in the glass-like transition temperature $\left(T_{g}\right)$ is associated to the immobilization of the polymer matrix by the presence of inorganic nanocomponents. There has been interaction areas proposed in which the polymer is immobilized, which increase in few degrees the softening temperature (23) and extends the useful life of the polymer, despite the fact that they may harden the material for its processing. The data shown in table 3 indicate that the glass-like transition of the nanocomposites prepared is reduced up to $20^{\circ} \mathrm{C}$; which means that the nanoparticles introduced do not make the PMMA rigid $\left(\mathrm{T}_{\mathrm{o}}=115^{\circ} \mathrm{C}\right)$. Table 3 also shows a higher drops of the values of $\mathrm{T}_{g}$ are produced with $10 \%$ in weight of the inorganic nanocompounds, specially for $\mathrm{SiO}_{2}$ where $\mathrm{T}_{\mathrm{g}}$ is reduced to a range between 80 and $101^{\circ} \mathrm{C}$.

Table 3. Results of the analyses DSC of nanocomposites

\begin{tabular}{|c|c|}
\hline Nanocomposite & $\mathrm{T}_{\mathrm{g}}\left({ }^{\circ} \mathrm{C}\right)$ \\
\hline $\mathrm{PMMA}_{\text {(acetone) }}-10-\mathrm{SiO}_{2}$ & 104 \\
\hline $\mathrm{PMMA}_{\text {(acetone) }}-20-\mathrm{SiO}_{2}$ & $112 ; 114$ \\
\hline $\mathrm{PMMA}_{\text {(isopropanol) }}-10-\mathrm{SiO}_{2}$ & $80 ; 84 ; 101$ \\
\hline $\mathrm{PMMA}_{\text {(isopropanol) }}-10-\mathrm{SiO}_{2}$ & $88 ; 104$ \\
\hline PMMA-10- $\mathrm{Al}_{2} \mathrm{O}_{3}$ & $98 ; 100$ \\
\hline PMMA-20- $\mathrm{Al}_{2} \mathrm{O}_{3}$ & $111 ; 112$ \\
\hline PMMA - $10-\mathrm{SiO}_{2}-\mathrm{Al}_{2} \mathrm{O}_{3}$ & $107 ; 112$ \\
\hline PMMA-20- $\mathrm{SiO}_{2}-\mathrm{Al}_{2} \mathrm{O}_{3}$ & $107 ; 110$ \\
\hline
\end{tabular}

The $\mathrm{T}_{\mathrm{g}}$ reduction may be caused by the plasticizer effect of the small inorganic molecules of the nanocomposite in the polymeric matrix, which easy the intramolecular movement of the lateral chains of the polymer. As reported in the biography, nanocomposites of alumina /PMMA have shown reductions of up to $25^{\circ} \mathrm{C}$ in the value of its $\mathrm{T}_{\mathrm{g}}$, with percentages of only $0.5 \%$ weight of alumina (14).

The stress status of the polymer around the nanoparticles may also influence the change of $\mathrm{T}_{\mathrm{g}}$ at a lower temperature. The zones of high and low mobility of the chain were previously reported (24) in a nanocomposite of PMMA; to this it may be attributed the different values of $T_{\mathrm{g}}$ for a the same sample in different evaluations. Besides the reduction of $\mathrm{T}_{\mathrm{g}}$, it is observed that the addition of nanoparticles produce a wider thermal transition, as observed in the DSC curve shown in figure 3.

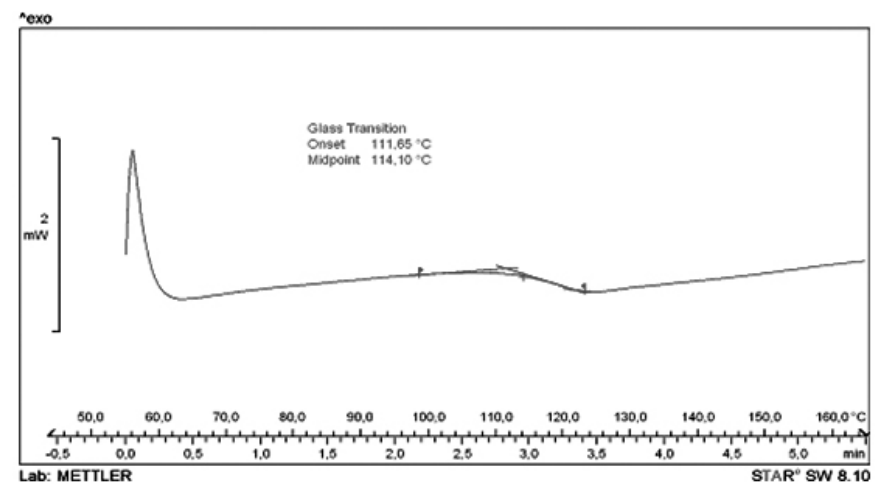

Fig. 3. DSC thermogram of $\mathrm{PMMA}_{\text {(isopropanol) }}-10 \%-\mathrm{SiO}_{2}$ nanocomposite.

Higher additions of the filler do not lead to reductions of the $\mathrm{T}_{\mathrm{g}}$ both for 
silica and alumina. Percentages of $20 \%$ in weight of the inorganic component, both of silica and alumina, increase the $\mathrm{T}_{\mathrm{g}}$ at similar values as pure PMMA. In this case, the high percentages of the nanocomponent act as a filler that reduce the free volume of the polymer and restrict the mobility of the chains increasing the $\mathrm{T}_{\mathrm{g}}$ value.

\section{CONCLUSIONS}

Nanocomposites have been synthesized PMMA-SiO, PMMA- $\mathrm{Al}_{2} \mathrm{O}_{3}$ and PMMA- $\mathrm{SiO}_{2}-\mathrm{Al}_{2} \mathrm{O}_{3}$. The nanocomposites were obtained by the incorporation of a filler by means of sol-gel to the polymer in solution. Morphologic, thermal and structural studies were performed to characterize the nanocomponents. The SEM and FT-IR studies support the formation of nanocomposites. Thermal studies show that the addition of nanoparticles $\mathrm{SiO}_{2}, \mathrm{Al}_{2} \mathrm{O}_{3}, \mathrm{SiO}_{2}-\mathrm{Al}_{2} \mathrm{O}_{3}$ modify the thermal degradation of PMMA.

\section{ACKNOWLEDGEMENTS}

The authors thank Dirección de Investigación (Universidad del Bío-Bío, Concepción) for their financial support.

\section{REFERENCES}

1. Wen, J.Y. and Wilkes, G.L., Chem. Mater., 8, 1667 (1996).

2. Sharp, K.G., Adv. Mater., 10, 1243 (1998).

3. Novak, B,M., Auerbach and Verrier, C., Chem. Mater., 6, 282 (1994).

4. Wang, S.H., Ahmad, Z. and Mark, J., Chem. Mater., 6, 943 (1994).

5. Shao, P.L., Mauritz, K.A. and Moore, R.B., Chem. Mater., 7, 192 (1995).
6. Komaneni, S.J., Mater. Chem., 2, 1219 (1992).

7. Giannelis, E.P., Adv. Mater., 8, 29 (1996).

8. Alexandre, M. and Dubois, P., Mater. Sci. Eng. Res., 28, 1 (2000).

9. Yoldas, B.E., Journal of Materials Science, 14, 1843-1849 (1979).

10. Gill, I., Chem. Mater., 13, 3404-3421 (2001)

11. Vyazovkin, S., Dranca, I., Ventilador, X.W. and Advíncula, R., J. Phys. Chem. B, 108, 11672 (2004)

12. Elim., H.I., Ji, W., Yuwono, A.H., Xue, J.M. and Wang, J., Appl. Phys. Lett., 82, 2691 (2003).

13. Laachachi, A., Cochez, M., Ferriol, M. , López-Cuesta, J.M. and Leory, E. Mater. Lett., 59, 36 (2005).

14. Ash, B.J., Siegel, R.W. and Schadler, L., J. Polym. Sci.: Part B., 42, 4371 (2004)

15. Lee, L.H. andChen, W.C., Chem. Mater., 13, 1137 (2001)

16. Manring, L.E., Sogah, D.Y. and Cohen, G.M., Macromolecules, 22, 4652 (1989).

17. Balamurugan, A., Kannan, S., Selvaraj, V. and Rajeswari, S., Trends Biomater. Artif. Organs, 18(1), 41 (2004)

18. Pickup, D.M., Mountjoy, G., Wallidge, G.W., Anderson, R., Cole, J.M., Newport, R.J. and Smith, M., J. Mater. Chem.., 9, 1299 (1999).

19. Madera D.L. and Rabinovich, E.M., Spectrosc., 43, 263 (1989)

20. Hwang, S.T., Hahn, Y.B., Nahm K.S. and Lee, Y.S., Colloids Surf. A Physicochem. Eng. Aspects, 259, 63 (2005)

21. Cheow, P-S., Liu, L. and Toh, C-S., Surf. Interface Anal., 39, 601 (2007)

22. Kondo, J., Ishikawa, H., Yoda, E., Wakabayashi, F. and Domen, K., J. Phys. Chem. B, 103, 8538 (1999).

23. Zhu, A.J., and Sternstein, S.S., Compos. Sci. Technol., 63, 113 (2003).

24. Tracht, U., Wilhelm, M., Heuer, A., Feng, H., Schmidt-Bohr, K. and Speiss, H.W., Phys. Rev. Lett., 81, 2727 (1998) 